\title{
Operations Dynamics of Gas Centrifugal Compressor: Process, Health and Performance Indicators
}

\author{
Helge Nordal Idriss El-Thalji \\ University of Stavanger, Norway, \{helge.nordal,idriss.el-thalji\}@uis.no
}

\begin{abstract}
Emerging technologies of Industry 4.0 have introduced novel ways of perceiving maintenance management, which has developed from being perceived as a "necessary evil" to become proactive with a holistic focusing on entire systems rather than single machines from Maintenance 3.0. In this context, the industry has begun to really appreciate the unique opportunities followed by system dynamics and simulation tools capabilities of representing the real world. However, maintenance management and performance are complex aspects of asset's operation that is difficult to justify because of its multiple inherent trade-offs. Although the majority areunanimous when it comes to the expected impact maintenance plays on company profitability, this is in most cases challenging to determine and quantify. Moreover, relevant literature is considered as limited, especially with regards to impact simulation of Maintenance 4.0. Therefore, this paper focuses on the supportive function system dynamics, and modeling and simulation tools can be of help to assess behavior and predicting the future outcome of Maintenance 4.0 in the era of Industry 4.0. This includes developing a conceptualized model that enables simulating the future expected behavior i.e. (un)availability and cost by implementing such a maintenance system. In this context, a centrifugal compressor with the function of exporting gas to Europe is applied as a case study.
\end{abstract}

Keywords: Industry 4.0 Architecture, system dynamics, maintenance management, impact simulation

\section{Introduction}

The perception of maintenance management has been highly influenced by several technological developments and evolved in from being a "necessary evil", "technical matter", "cost-cutting contributor", "profit contributor" until it today is perceived as a "cooperative partnership" that can potentially add value to the business (Pintelon and Parodi-Herz, 2008). Moreover, maintenance strategies have developed rapidly over the last three industrial revolutions from being reactive, preventive, predictive and finally to become proactive and holistic (focus on entire system rather than single machine) (Alsyouf, 2007). Those strategic changes lead to several maintenance programs e.g. reliability centered maintenance (RCM), condition based maintenance $(\mathrm{CBM})$, and total productive maintenance (TPM) (Pintelon and Parodi-Herz, 2008).

Maintenance is often associated with high cost allocated in the phase of an asset's operation and maintenance $(\mathrm{O} \& M)$. In fact, the cost of maintenance is determined as sector dependent and varies all from 15 to 70 percent of total production cost (Bevilacqua and Braglia, 2000; Mobley, 2002; Gräber 2004). Moreover, several authors state that maintenance management is often attributed to poor planning and decision support resulting into inefficiency and waste of both cost and resources (Wireman, 1990; Noemi and William, 1994; Mobley, 2002; Wireman, 2004; Bardey, 2005). However, maintenance management and performance are complex aspects of asset's operation that is difficult to justify because of its multiple inherent trade-offs. Therefore, the expected or estimated impact of maintenance is often challenging to determine and quantify. Nevertheless, several literature are in fact unanimous when it comes to the expected impact maintenance plays on company profitability, supporting current perception of maintenance as a "cooperative partnership" (Komonen, 2002; Alsyouf, 2007; Maletič et al., 2012; Erguido et al., 2017; Colledani et al., 2018; Olde Keizer et al., 2018).

The importance of facilitating simulating the expected impacts of emerging technologies in the context of Industry 4.0 and its associated concepts has never been as important as it is today. It exists a great optimism of what the expected impact Industry 4.0 will bring to the oil and gas sector (O\&G). (World Economic Forum, 2017) states that predictive maintenance and operations optimization are the two main technologies that will have the highest industrial and societal impacts in this specific sector, by e.g. reducing maintenance costs by $20 \%$ and operational downtime by $5 \%$ (mainly due to predictive maintenance). However, nowadays, only one single percent of data originating from 30000 sensors located on a traditional oil rig is exploited. Moreover, this single percentage is in most cases utilized with regards to control and detection of operational anomalies (Manyika et al., 2015) and not for optimization and prediction in which World Economic Forum (2017) highlights as the most beneficial impact. 
Moreover, as the cumulative maintenance expenditures concerning offshore installations with the design life of 30 years can reach up to $£ 2$ Billion, and often more, as life-extending measures are commonly adopted to enable operating such facilities over its designed lifetime (Register, 2017), there exist a great potential of reducing the associated cost by $£ 400$ Million, given that a $20 \%$ reduction in maintenance cost as presented by World Economic Forum (2017) is reasonable. Hence, the potential impacts of adopting an intelligent maintenance system in Industry 4.0 is considered as tremendous. However, estimating these specific impacts of predictive maintenance and operations optimization require techniques with growing complexity and capabilities. Therefore, simulation is considered as the key enabling technology as highlighted by (Oesterreich and Teuteberg, 2016) that may facilitate forecasting impacts of e.g. intelligent maintenance in Industry 4.0 in the context of predictive maintenance and operations optimization.

Several studies have adopted the methodology of system dynamics and investigated the opportunities of developing a model that enables simulating the impact of maintenance or internet of things (IoT) with respect to output variables such as e.g. companies' availability, cost and profit (Jokinen et al., 2011; Endrerud et al., 2014; Manyika et al., 2015; Hussain et al., 2016; Linnéusson et al., 2016; Qu et al., 2016; Linnéusson et al., 2018; McKee et al., 2017; Marshall, 2015; Jalali et al. 2017; Markus et al., 2018). Regardless, the authors identify two research gaps: (1) none of the existing models enables simulating the causal relationship that is present in the maintenance phase of a centrifugal compressor applied to export gas in the O\&G industry and (2) none of existing literature facilitate simulation of intelligent maintenance architecture based on Industry 4.0 requirements. Hence, the purpose of this paper aims to develop a conceptualized model that enables simulating both the behavior of the associated compression system applied in gas export and the expected impacts introduced through the implementation of an intelligent maintenance system, by adopting the methodology of systems dynamics (SD) and simulation.

The remainder of this paper is organized as follows. Section 2 presents some relevant theory regarding process modeling and industrial simulation. Then, the case study of the centrifugal gas export compressors is investigated in Section 3. Section 4 is dedicated to the presentation of the conceptualized model developed in which enables simulating the behavior of the case study and the expected impact of implementing intelligent maintenance in the era of Industry 4.0. At last, Section 5 provides some concluding remarks.

\section{Process Modeling and Industrial Simulation}

Industry 4.0 and associated emerging technologies of cyber-physical systems (CPS), internet of things (IoT), big data, and cloud computing (including diagnosis and prognosis) are expected to play a vital role in companies future competitiveness and sustainability. However, determining the future benefits of adopting such technologies is of vital importance. Therefore, modelbased representations i.e. process modeling and industrial simulation approaches e.g. system dynamics have become a highly embraced tool with its growing complexity and capabilities (McKee et al., 2017). This includes tools such as e.g. discrete event simulation (e.g. Arena), system dynamics (e.g. Vensim), and multi simulation methods (e.g. Anylogic and Numerus (Nova)), which all are frequently applied by industries to facilitate modeling and simulation of complex processes to perceive their nonlinear characteristics, either in the phase of early design, operation and maintenance, or late in decommissioning. The benefit of adopting process modeling and industrial simulation relates to its unique way of representing scenarios that are as close as possible to the real world. In this process, causal relationships are investigated and described in details, yielding an understanding of system behavior.

The literature describes the application of process modeling and industrial simulation in order to enhance asset operation. This is especially interesting i.e. maintenance and its impact on companies' availability, cost, and profit. In this context, some of the most wellknown simulation models are presented in Table 1, considering their associated influencing variables, rates, and estimated benefits. Moreover, these well-known models constitute a supportive function when developing the conceptualized model presented in this paper. In fact, the model of (Jokinen et al., 2011) is especially interesting by its classification of failures (critical, degraded, and incipient) and its description of detection rate w.r.t sensor technology and maintenance management.

In this paper, the authors intend to adopt the approach of system dynamics to investigate and describe the case study and successively aid developing a model that enables simulating the causal relationship(s) associated with the operation of the case study. 
Table 1. Summary of four of the most well-known simulation models.

\begin{tabular}{|c|c|c|c|}
\hline & Influencing variables & Rates & Estimated benefits \\
\hline $\begin{array}{l}\text { (Linnéusson } \\
\text { et al., 2018) }\end{array}$ & $\begin{array}{l}\text { - Number of repair workers and maintenance } \\
\text { engineers } \\
\text { - Goal and fraction of PM and CBM based on root } \\
\text { cause analysis } \\
\text { - Inspection interval } \\
\text { - Scheduled and unscheduled repairs } \\
\text { - Backlog of PM and CBM } \\
\text { - Equipment health }\end{array}$ & $\begin{array}{l}\text { - Take down and break down rate } \\
\text { - Mean Time To Failure (MTTF) }\end{array}$ & $\begin{array}{l}\text { - Availability } \\
\text { - Maintenance cost } \\
\text { - Acc. maintenance } \\
\text { budget margin } \\
\text { - Acc. company } \\
\text { result }\end{array}$ \\
\hline $\begin{array}{l}\text { (Jambekar, } \\
2000 \text { ) }\end{array}$ & $\begin{array}{l}\text { - Throughput pressure } \\
\text { - Planned throughput } \\
\text { - Preventive maintenance }\end{array}$ & $\begin{array}{l}\text { - Equipment and labor force utilization } \\
\text { - Breakdown }\end{array}$ & $\begin{array}{l}\text { - Process quality } \\
\text { - Process reliability }\end{array}$ \\
\hline $\begin{array}{l}\text { (Zuashkiani, } \\
\text { et al., 2011) }\end{array}$ & $\begin{array}{l}\text { - Resource shift to reactive maintenance } \\
\text { - Production } \\
\text { - No. of repairmen allocated to reactive } \\
\text { maintenance } \\
\text { - No. of repairmen left to proactive maintenance } \\
\text { - Reactive maintenance } \\
\text { - Collateral damage }\end{array}$ & $\begin{array}{l}\text { - Accumulated defects } \\
\text { - Breakdown } \\
\text { - Downtime due to proactive maintenance } \\
\text { - Pressure on production } \\
\text { - Take down rate for proactive maintenance } \\
\text { - Downtime due to reactive maintenance } \\
\text { - No equipment time for PM }\end{array}$ & - Plant OEE \\
\hline $\begin{array}{l}\text { (Honkanen, } \\
\text { 2004) }\end{array}$ & $\begin{array}{l}\text { - Working components } \\
\text { - Components that are either failing soon, under } \\
\text { repair, or have failed due to aging and infant life. } \\
\text { - Working and failing components to be CBM } \\
\text { maintained. } \\
\text { - Components under maintenance }\end{array}$ & $\begin{array}{l}\text { - (Net, Effective and Total) operating time } \\
\text { - (Net) availability } \\
\text { - (Scheduled) downtime } \\
\text { - Speed and defect losses } \\
\text { - Work start rates of repair, PM work, and CBM } \\
\text { work. }\end{array}$ & $\begin{array}{l}\text { - Availability } \\
\text { - Performance } \\
\text { - Quality }\end{array}$ \\
\hline $\begin{array}{l}\text { (Jokinen et } \\
\text { ol., 2011) }\end{array}$ & $\begin{array}{l}\text { - Spare part management } \\
\text { - Maintenance management (e.8. target and } \\
\text { active tasks related to preventive and corrective } \\
\text { maintenance) }\end{array}$ & $\begin{array}{l}\text { - Time } \\
\text { - Increased degradation with time } \\
\text { - New components and worn out components } \\
\text { - Failures (incipient, degraded, and critical) }\end{array}$ & $\begin{array}{l}\text { - Availability } \\
\text { - Maintenance cost }\end{array}$ \\
\hline
\end{tabular}

\section{Case Study}

The case study in this investigation is a compression system with the primary function of enabling transporting sales gas (processed natural gas) through subsea pipelines by dynamically compressing it from 62 to $185 \mathrm{barg}$ - thus, decisive as it determines whether the end users receive their booked gas or not. The compression system comprises four identical compressor trains, which each includes an electric motor, gearbox, and two centrifugal compressors arranged in series. In operation, only two out of the four compressor trains are in operation and sharing loads. Hence, two trains are at any time functioning as redundancy in cold standby (Wang, 2009; Tan, 2011). A cold standby system is a system that does not deteriorate or includes economic dependence of the operation.

Moreover, each compressor train includes 45 sensor signals related to condition monitoring: 26 sensor signals of single sensor signal that monitors vibration in terms of velocity. The data generated by the different sensors are in most cases exploited through trending. Therefore, it is of interest to investigate the behavior of the respective compression system (as a decisive part of the transportation system) and how a potential implementation of an intelligent maintenance system in the era of Industry 4.0 may impact the operation/ transportation in terms of availability and cost. To do so, the methodology of system dynamics and modeling and simulation is adopted. In order to manage to develop a simulation model that allows simulating the expected impacts, several processes must be studied at first:

\section{Gas production/compression scenario (normal} loading, seasonal demand): The need for gas compression is highly dependent on the season as the consumers mainly utilize the gas for heating and cooking at households (in addition to industrial consumption). Thus, end-user demand is peaking at winter-season when the need for heating is at its greatest. From a maintenance perspective, this will obviously play an important role when it comes to planning as a specific action will affect the operational (un)availability differently.

2. Planned maintenance scenario (planned schedule timeline): Equipment vendor usually provides customers with recommendations of preventive maintenance action throughout the equipment's life cycle. These are often based on either time in operation or numbers of cycles. Clearly, this will have an impact on planned unavailability and maintenance cost, and must, therefore, be considered in the context of intelligent maintenance and maintenance decision support (maintenance optimization).

3. Failure growth scenarios: The occurrence of symptoms of failure, or even failure, rises demand for necessary future action. The (symptom of) failure will 
impact the operation differently based on its characteristics i.e. location, size, and severity. This is highly associated with maintenance management and the application of condition monitoring technologies including sensor technology, diagnosis, prognosis, which determines detection stage and thereby the criticality of the degradation and urgency for repair along with its cost.

4. Fault detection scenario: The introduction of Industry 4.0 has brought novel opportunities in maintenance management, especially considering cloud computing including diagnosis and prognosis. In more detail, maintenance 4.0 differentiates from maintenance 3.0 by moving its focus from the traditional enterprise level to become more holistic comprising the asset-level and between asset and enterprise-level. Therefore, it is of interest to simulate the difference between three monitoring scenarios in terms of impact: (1) compressor without CMS, (2) compressor with CMS (maintenance 3.0), and (3) compressor with maintenance 4.0. In this context, it is expected that the detection rate related to condition monitoring increases and the cost related to the level of repair is reducing, respectively.

5. Fault prevention scenario (by the control system): The objective of intelligent maintenance is to enhance right maintenance to be executed at the right time. This could e.g. include performing temporary maintenance actions that extend the remaining useful lifetime (RUL) estimate, which enables delaying the need for executing the required maintenance action from the diagnosis - thus, gaining opportunistic benefits. This comprises, for instance, the ability to plan the maintenance work for next opportunistic interval e.g. low production season or no-production days. Additionally, preventing the needs for corrective (maintenance) measures, which is commonly known as costly in comparison to other maintenance strategies.

6. Fault prevention scenario (by maintenance action): Accurate health assessments (diagnosis) and remaining useful life (RUL) estimations enable developing detailed work orders including spare part management, required human resources, and expected execution time. This improves the maintenance supportability and enhances the maintenance action's successfulness ensuring that the right maintenance action is conducted.

7. Maintenance performance: It is clear that execution of diagnosis will pose a beneficial impact of the maintenance performance since it enables assessing the current health status of the equipment and by this pinpoint the exact degradation mechanism. Thus, if the diagnosis e.g. reveals degradation of outer racing of a roller bearing, the maintenance personnel can plan down to the smallest details on how to maintain this in the best way. Hence, enhancing the improved performance of the maintenance action that is measurable through e.g. the mean-time-to-repair (MTTR).

8. Spare Part Management: The capabilities of prognostics is expected to impact the cost of inventory since it reduces the need for having spare parts in stock as it is possible to forecast at what time the different spare parts are required in advance.

Table 2. Summary of the related case study scenarios with associated potential estimated impact.

\begin{tabular}{|l|l|}
\hline Scenarios Affecting Transportation & Potential Estimated impact \\
\hline $\begin{array}{l}\text { Gas production/compression scenario } \\
\text { (normal loading, seasonal demand) }\end{array}$ & Availability, performance \\
\hline Planned maintenance scenario & Planned Unavailability, planned maintenance cost \\
\hline Failure growth scenario & Unplanned Unavailability, unplanned maintenance cost, equipment degradation \\
\hline Fault detection scenario & $\begin{array}{l}\text { Saving losses (unplanned maintenance cost, cost related to the level of repair (since the fault } \\
\text { was detected before the whole system was damaged), etc.) }\end{array}$ \\
\hline Fault prevention scenario & $\begin{array}{l}\text { Gain opportunistic benefits e.g. able to plan the maintenance work for next opportunistic } \\
\text { interval e.g. low production season or no-production days. }\end{array}$ \\
\hline Maintenance performance scenario & $\begin{array}{l}\text { Operational availability and unavailability (e.g. MTTR), cost of inventory, required human } \\
\text { resources }\end{array}$ \\
\hline Spare part management scenario & Cost of inventory \\
\hline
\end{tabular}

\section{The Conceptualized Model}

The prime objective of implementing intelligent maintenance architecture based on Industry 4.0 is to facilitate optimizing existing maintenance schedule. Although, the definition of "optimization" (Diwekar, 2008) can be interpreted ambiguously, it is in this context referred to as the mathematical concept that determines the optimal solution to a function comprising several different input and output variables such as e.g. gas price, cost, spare part management, operational (un)availability, and resources required and available. The most optimal solution based on the multiple variables is dependent on their weighting that may vary from time to time, under different circumstances. Resultantly, the optimization process shall ensure that the right maintenance takes place at the right time. 
The conceptualized model developed to enable simulating this optimum maintenance action is based on maintenance theory from the literature review, systems dynamics (Vensim), the well-known simulation models presented in Table 1, and the scenarios extracted from the case study. As seen from the conceptualized model presented in Figure 1, it can be decomposed into four sub-models: (1) production, (2) maintenance management, (3) CBM, and (4) equipment degradation. In general, production (sub-model 1) is similar to any commodity, highly associated with the logic of supply and demand comprising variables such as e.g. politics, regulations, wealth, technology, population, and substitutes. Briefly, this is, in turn, determining the price of the commodity and thereby the revenue of the company. Moreover, the production is restricted by the availability of resources (hydrocarbons) and the associated overall equipment efficiency (OEE) that is governed by specific maintenance management (submodel 2). The most important content of maintenance management is to optimize the existing maintenance schedule. This includes detecting failures (diagnosis), predicting the future development of the degradation and its impact on the system (prognosis), in which enables developing a detailed maintenance plan that identifies the best opportunistic window to perform the required action that improves availability and thus minimizes unavailability.

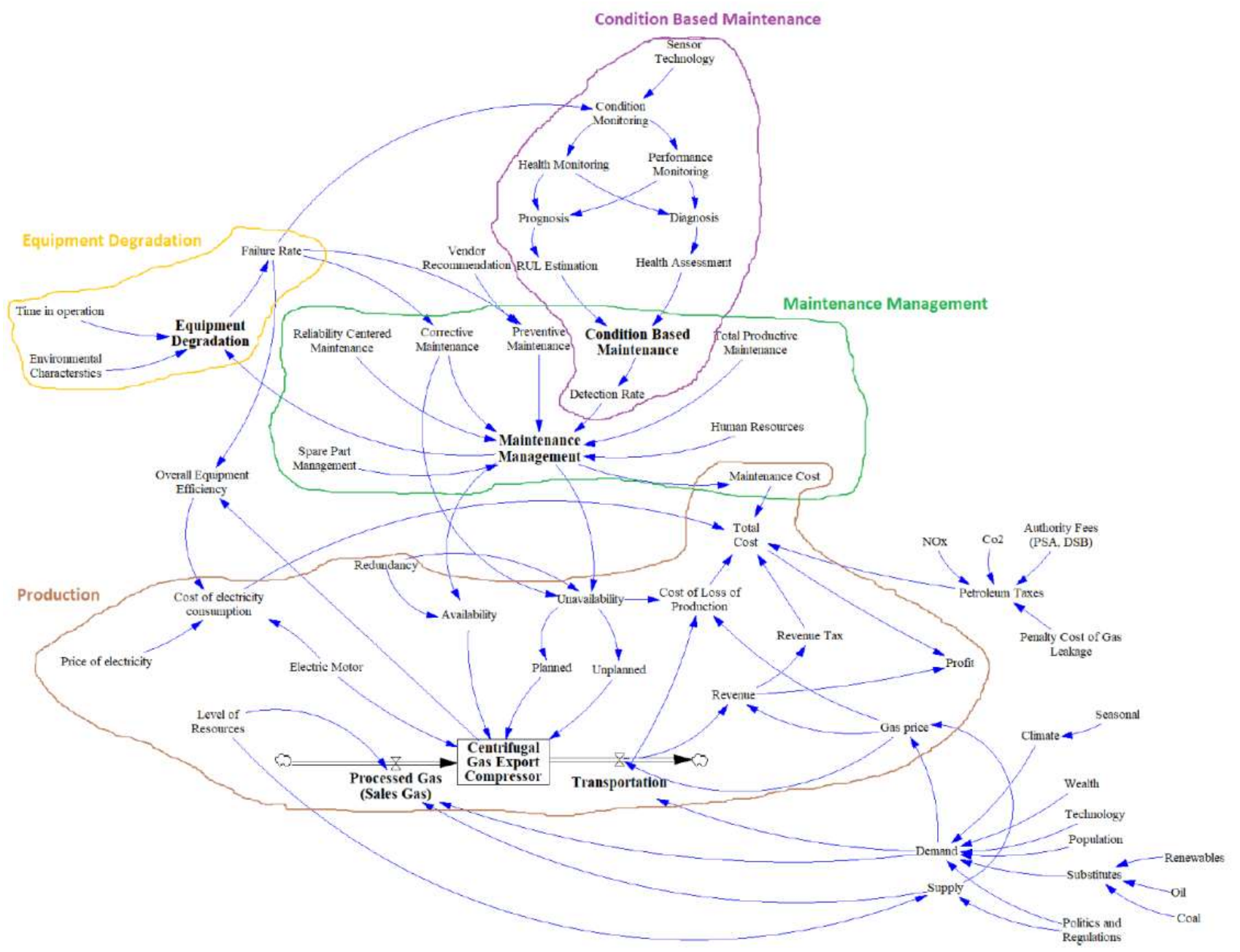

Figure 1. The conceptualized simulation model developed with associated sub-models.

CBM (sub-model 3) is all about monitoring performance and health data through sensor technology in order to detect (diagnosis), predict (prognosis), and extend the RUL estimate by prolonging the time to when the specific maintenance action is required. In this context, failure modes are classified into critical, degraded, and incipient failure modes. Traditionally, critical failure modes are detectable through performance monitoring (variables related to the process such e.g. pressure, temperature, and flow), while health parameters can improve the detection rate of critical failure modes and may even comprise certain degraded failure modes by combining performance and health parameters in terms of multivariate analyses of big data.

In order to detect degradation mechanisms early, awareness of equipment degradation (sub-model 4) affected by certain characteristics such as e.g. 
environment (weather and climate) and operation (rpm and loading) is of high importance as it is connected with the equipment's failure rate. Moreover, such awareness supports achieving an understanding of equipment behavior along with responding to the results from the diagnosis and prognosis by e.g. prolonging the time to required maintenance action.

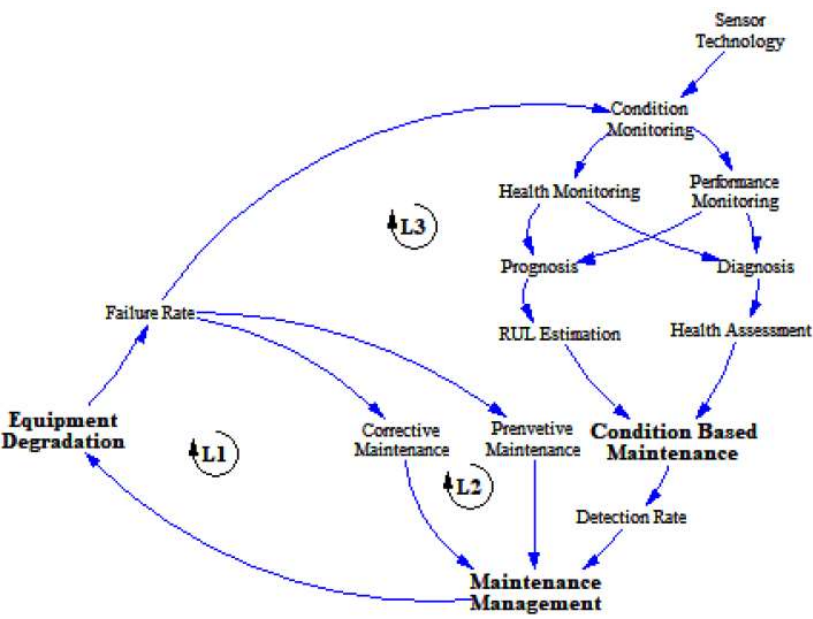

Figure 1. Three different causal loops identified by the conceptualized simulation model.

In final, Figure 2 shows the three causal loops identified in the conceptual model, which is the basic benefit of systems dynamic to manage (balancing or reinforcement). To summarize, the conceptualized simulation model shows the interaction between the different maintenance strategies affecting maintenance management. Furthermore, this plays a vital role for the estimated impacts i.e. availability and cost efficiency (as shown in Figure 1). It is clear from Figure 2 that the causal loop of CBM (L3) yields the greatest impacts and thus the objective of maintenance management, and vice versa for the causal loop regarding corrective maintenance (L1). However, in order to estimate the specific impacts, the capabilities of detecting the different failure modes associated with the system through sensor technology (parameter monitoring) and data analyses (diagnosis and prognosis) must be investigated.

\section{Conclusions}

Industry 4.0 and associated emerging technologies of CPS, IoT, big data, and cloud computing (including diagnosis and prognosis) are expected to play a key role in companies' future competitiveness and sustainability. However, determining these future benefits of adopting such technologies is of vital importance. Therefore, process modeling and industrial simulation approach e.g. system dynamics have become a highly embraced tool with its growing complexity and capabilities to facilitating perceiving nonlinear characteristics of complex processes.
Literature describes the importance of having a rigid maintenance management, which is reflected in associated impacts such as e.g. availability and cost estimations. Moreover, based on the growing complexities and capabilities of system dynamics, simulation is frequently adopted to estimate such impacts. However, maintenance management and performance are complex aspects of asset's operation that is difficult to justify because of its multiple inherent trade-offs. Although the majority are unanimous when it comes to the expected impact maintenance plays on company profitability, this is in most cases challenging to determine and quantify.

The conceptualized model developed in this paper demonstrates three causal relationships between different scenarios and its potential estimated impacts related to a case study concerning a centrifugal compressor that is applied to transport natural gas. The three causal loops (corrective maintenance, preventive maintenance, and CBM) shows the importance of having rigid maintenance management and its impact on company availability and cost. However, in order to quantify these specific impacts, the capabilities of detecting the different failuremodes associated with the system through sensor technology (parameter monitoring) and data analyses (diagnosis and prognosis) must be investigated. Moreover, it is expected that implementation of maintenance 4.0 will not only aid for improved operational impacts such as those two mentioned but also enhance the asset's HSE. This is described rather narrowly in the report. Nevertheless, HSE is of greatest importance but is excluded as it is challenging to quantify and simulate such intangible aspects of the operation.

\section{References}

I. Alsyouf. The role of maintenance in improving companies' productivity and profitability. International Journal of Production Economics, 105(1): 70-78, 2007.

D. Bardey. To maintain or not maintain? what should a riskaverse decision maker do?. J Qual Maint Eng, 11: 115120, 2005.

M. Bevilacqua and M. Braglia. The analytic hierarchy process applied to maintenance strategy selection. Reliab. Eng. Syst. Saf., 70(1): 71-83, 2000.

M. Colledani, M. C. Magnanini, and T. Tolio. Impact of opportunistic maintenance on manufacturing system performance. In CIRP Annals, 2018.

U. Diwekar. Introduction to Applied Optimization. In Springer Science \& Business Media. Berlin, Germany. 22, 2008.

O.-E. V. Endrerud, J. P. Liyanage, and N. Keseric. Marine logistics decision support for operation and maintenance of offshore wind parks with a multi method simulation model. In Proceedings of the 2014 Winter Simulation Conference, 2014.

A. Erguido, A. Crespo Márquez, E. Castellano, and J. F. Gómez Fernández. A dynamic opportunistic mainte- 
nance model to maximize energy-based availability while reducing the life cycle cost of wind farms. Renewable Energy, 114: 843-856, 2017.

U. Gräber. Advanced maintenance strategies for power plant operators - introducing inter-plant life cycle management. In 29th MPA Seminar in the series Safety and Reliability of Pressure Components Stuttgart, Int. J. Press. Vessels Pip., 81 10-11: 861-865, 2004.

T. Honkanen. Modelling Industrial Maintenance Systems and the Effects of Automatic Condition Monitoring. Master of Science, Helsinki University of Technology Information and Computer Systems in Automation, 2004.

Y. M. Hussain, S. Burrow, L. Henson and P. Keogh. Benefits Analysis of Prognostics \& Health Monitoring to Aircraft.

Maintenance using System Dynamics. In EUROPEAN CONFERENCE OF THE PROGNOSTICS AND HEALTH MANAGEMENT SOCIETY, 2016.

M. S. Jalali, J. P. Kaiser, M. Siegel, and S. Madnick. The Internet of Things (IoT) Promises New Benefits - and Risks: A Systematic Analysis of Adoption Dynamics of IoT Products. W. P. C. 2017-15, Cybersecurity Interdisciplinary Systems Laboratory (CISL), Sloan School of Management, Massachusetts Institute of Technology Cambridge, 2017.

A. B. Jambekar. A systems thinking perspective of maintenance, operations, and process quality. Journal of Quality in Maintenance Engineering, 6(2): 123-132, 2000.

T. Jokinen, P. Ylén, and J. Pyötsiä. Dynamic Model for Estimating the Added Value of Maintenance Services, Technical research centre of Finland, VTT, 2011.

K. Komonen. A cost model of industrial maintenance for profitability analysis and benchmarking. Int. J. Production Economics, 79: 15-31, 2002.

G. Linnéusson, A. $\mathrm{Ng}$, and $\mathrm{T}$. Aslam. Investigating Maintenance Performance: A Simulation Study. In 7th Swedish Production Symposium, Lund, Sweden, October 25-27, 2016.

G., Linnéusson, A. H. C. Ng, and T. Aslam. Quantitative analysis of a conceptual system dynamics maintenance performance model using multi-objective optimisation. Journal of Simulation, 12(2): 171-189, 2018.

G. Linnéusson, H. C. A. Ng and T. Aslam. Towards strategic development of maintenance and its effects on production performance by using system dynamics in the automotive industry. International Journal of Production Economics 200: 151-169, 2018.

D. Maletič, M. Maletič, B. Al-Najjar and B. Gomišček. The role of maintenance regarding improving product quality and company's profitability: A case study. IFAC Proceedings Volumes 45(31): 7-12, 2012.

J. Manyika, M. Chui, P. Bisson, J. Woetzel, R. Dobbs, J. Bughin and D. Aharon. THE INTERNET OF THINGS: MAPPING THE VALUE BEYOND THE HYPE EXECUTIVE SUMMARY, McKinsey \& Company, 2015.

A. Markus, A. Marques, G. Kecskemeti, and A. Kertesz. Efficient Simulation of IoT Cloud Use Cases. Autonomous Control for a Reliable Internet of Services, pages 313-336, 2018.

P. Marshall. System dynamics modeling of the impact of Internet-of-Things on intelligent urban transportation. In
Regional Conference of the International Telecommunications Society (ITS), Los Angeles, CA, 25-28 October, 2015, International Telecommunications Society, Los Angeles.

D. W. McKee, S. J. Clement, X. Ouyang, J. Xu, R. Romanoy and J. Davies. The Internet of Simulation, a Specialisation of the Internet of Things with Simulation and Workflow as a Service (SIM/WFaaS)). In 2017 IEEE Symposium on Service-Oriented System Engineering (SOSE): 47-56, 2017.

R. Mobley. An Introduction to Predictive Maintenance. 2002.

M. P. Noemi and L. William. Maintenance Scheduling: Issues, Results and Research Needs. International Journal of Operations \& Production Management 14: 47-69, 1994.

T. D. Oesterreich and F. Teuteberg. Understanding the implications of digitisation and automation in the context of Industry 4.0: A triangulation approach and elements of a research agenda for the construction industry. Computers in Industry, 83: 121-139, 2016.

M. C. A. Olde Keizer, R. H. Teunter, J. Veldman and M. Z. Babai. Condition-based maintenance for systems with economic dependence and load sharing. International Journal of Production Economics, 195: 319-327, 2018.

L. Pintelon and A. Parodi-Herz. Maintenance: An Evolutionary Perspective. In Complex System Maintenance Handbook. Springer, London, Springer Series in Reliability Engineering, 2008.

T. Qu, M. Thürer, J. Wang, Z. Wang, H. Fu, C. Li and G. Q. Huang. System dynamics analysis for an Internet-ofThings-enabled production logistics system. International Journal of Production Research 55(9): 2622-2649, 2016.

L. S. Register. Reducing the maintenance burden, 2017. Retrieved 20th of March, 2019, from https:/www.offshoreenergytoday.com/reducing-themaintenance-burden.

L. Tan, J. Yang, Z. Cheng and B. Guo. Optimal replacement policy for cold standby system. Chin. J. Mech. Eng 24 (2): 316-322, 2011.

L. Wang, E. Zheng, Y. Li, B. Wang, J. Wu. Maintenance optimization of generating equipment based on a conditionbased maintenance policy for multi-unit systems. In Chinese Control and Decision Conference (CCDC 2009), IEEE: 2440-2445, 2009.

T. Wireman. World class maintenance management, 1990.

$\mathrm{T}$. Wireman. Benchmarking best practices in maintenance management. In Industrial Press, 2004. World Economic Forum. Digital Transformation Initiative Oil and Gas Industry, 2017.

A. Zuashkiani, H. Rahmandad and A. K. S. Jardine. Mapping the dynamics of overall equipment effectiveness to enhance asset management practices. Journal of Quality in Maintenance Engineering, 17(1): 74-92, 2011. 